\title{
Sur la perte de vitalité du diminutif en français
}

\author{
Samuel BIDAUD \\ Université de Bourgogne \\ samuel.bidaud@u-bourgogne.fr
}

\begin{abstract}
RESUMEN
El presente artículo trata el tema de la pérdida de vitalidad del diminutivo en lengua francesa, muy inferior a otras lenguas románicas. El autor le presta especial atención al uso de este morfema en el español, donde sí que presenta una mayor frecuencia. El diminutivo del francés se habría lexicalizado y habría perdido su vitalidad.
\end{abstract}

Palabras clave: diminutivo, lengua francesa, lengua española, lexicalización

[Recibido, diciembre 2010; aprobado, marzo 2011]

On the loss of vitality of diminutive in French

\begin{abstract}
This paper considers the subject of the vitality of diminutives in French, lower than in other Romance languages. The author pays special attention to the use of this morpheme in Spanish, where it does have a higher frequency. The diminutive in French would be lexicalised and therefore it would have lost it vitality.
\end{abstract}

Keywords: Diminutive, French language, Spanish language, lexicalization 
1.

Le fait a souvent été relevé que le diminutif créatif était d'une vitalité très faible en français par rapport à l'ensemble des autres langues romanes (Hasselrot 1972: 11). En effet, là où l'espagnol utilise les diminutifs d'une manière abondante, le français, lui, préfère la tournure analytique avec l'adjectif « petit » et ne conserve de morphème diminutif presque qu'à l'état lexicalisé. Certes, certains suffixes comme « ette » peuvent encore être employés de manière créative, mais le nombre de créations spontanées auxquelles un tel diminutif donne lieu est très limité.

Rappelons simplement que le diminutif peut donner lieu à des créations qui modifient presque toutes les parties du discours dans les langues romanes qui ne sont pas le français : outre des substantifs (esp. «tarrito de fresa », « mujercita », « Lolita »), des adjectifs (port. « uma rapariga bonitinha », it. « E a far che? Poverina! », Goldoni, Gli amori di Zelinda e Lindoro), des adverbes (port. «Basta ouvir as protestas que eles murmuram, baixhino, entre si e Deus, para crer que esses dous corações podem ficar separados pelo mar, mas que o amor os uniu moralmente e eternamente », Machado de Assis, Letra vencida, esp. « « Hacemos viajes rapidito», refieren los avisos colocados con spray», El Universal), le diminutif peut modifier des pronoms, par exemple dans l'espagnol « todito» (« Con los potes de leche eran baratos, todito», oral, Venezuela), « estito » ( « Primero he aprendido piano, mi profesor en Potosí me ha enseñado, luego he aprendido acordeón, después guitarra, después charango, mandolina, y ahora toco estito », Los Tiempos, 2007), « esito » (José G. Mendoza (2008: 230) relève par exemple la phrase suivante : «Esito nomás sería » en espagnol de Bolivie), dans le roumain populaire «mătăluță » (diminutif péjoratif de «matale », forme de politesse), « cutărică » (diminutif péjoratif de «cutare », « tel »), etc. Le diminutif peut également modifier de manière créative des interjections ( ¿ ¿Vas a llamarnos de verdad, Nelson? - Clarito que sí! », Juan Madrid, Flores, el gitano), et nous pourrions également rapprocher certaines formules des interjections, par exemple « Hasta luego », pour lequel nous avons relevé le diminutif « ¡Hasta lueguito!» (Mendoza 2008: 230), des gérondifs (outre le très souvent cité «callandito », la Real Academia (2009) relève également " andandito » ou " paseandito ») ou même des verbes conjugués (ainsi dans le portugais «Estazinha », "Elle est, avec un diminutif sur le verbe conjugué » (cité par Hasselrot 1957: 277)).

En tout cas, d'un point de vue strictement quantitatif, la vitalité de la suffixation diminutive française est quasi nulle par rapport à l'espagnol, au portugais ou à l'italien. Le phénomène est d'autant plus curieux que, historiquement, le français a bien eu recours à la suffixation diminutive créatrice jusqu'à l'âge classique. Le diminutif est très présent à la Renaissance en France aussi bien qu'en Espagne. Ouvrons Ronsard ou un autre poète de l'époque, et l'on y trouvera de nombreux diminutifs, depuis les "nymphettes » jusqu'aux " mignonnettes » souvent citées. La langue parfaite est alors celle qui a le plus de procédés expressifs d'après Hasselrot, qui peut écrire à propos d'un grammairien comme 
Henri Estienne que, « tout enivré qu'il est de chauvinisme linguistique », ce dernier « n'exagère pas, ou guère, quand il prétend que le français peut se mesurer avec l'italien, et même le surclasser, en ce qui concerne les diminutifs, qui sont pour lui une des principales «mignardises » de sa langue maternelle » (Hasse1rot 1957: 213-214).

Ces faits rappelés, il est d'autant plus surprenant de constater qu'à l'âge classique les diminutifs disparaissent brusquement. On passe en effet d'un état de langue où le diminutif est extrêmement présent à un état où l'on n'en observe presque plus. Même chez des auteurs qui imitent parfois le style paysan, comme Molière au dix-septième siècle et Marivaux au dix-huitième siècle, il ne nous semble pas avoir relevé de diminutifs sinon très occasionnellement. Comment expliquer ce qu'il convient bien d'appeler, sinon une disparition, du moins une perte de vitalité considérable du suffixe diminutif à l'âge classique? Alors que le diminutif continue à être très présent dans les autres langues romanes, le français, au contraire, perd ce morphème. A cela nous avancerons plusieurs éléments d'explication. Le premier, historiquement déterminé, aura pour but d'expliquer la raison de la disparition du diminutif précisément à l'âge classique.

En effet, cette disparition est à notre avis une conséquence du paradigme logique qui prévaut à cette époque en France parmi les grammairiens et qui consiste à voir dans le français une langue miroir de l'esprit humain, une langue logique, qui reflète l'ordre de la pensée et qui est la langue de la science par excellence. Or, une telle conception de la langue, qui est reprise par bien des grammairiens de cette époque, et qui a eu beaucoup d'influence sur les écrivains, implique que le diminutif, morphème approximatif affectif, va à l'encontre du caractère « scientifique » de la langue française. On verra que cet argument est plus considérable qu'il ne semble au premier abord, et que les grammairiens de cette époque reflétaient plus largement un certain état d'esprit qui peut réellement fonctionner comme un élément d'explication de la disparition du diminutif du français littéraire. Lorsqu'on sait que c'est ce français littéraire qui prévaut dans l'enseignement, on imagine quelles ont pu en être les conséquences.

Mais au-delà de cela, nous voyons un deuxième élément d'explication de la perte de vitalité du diminutif en français. Cet élément est le suivant : le français est une langue essentiellement analytique, et est d'un degré d'analyticité beaucoup plus fort que l'espagnol, l'italien ou le portugais, d'où l'utilisation d'un adjectif subduit, « petit », pour remplacer le diminutif. Nous montrerons donc, à travers plusieurs exemples, que les autres langues romanes sont d'un degré analytique moins avancé que le français, ou, dit autrement, restent plus synthétiques $^{1}$

${ }^{1}$ Cette idée nous a été suggérée lors d'une conversation par Philippe Monneret. 


\section{L'âge classique et l'épistémè de la représentation}

Le titre que nous avons donné à ce paragraphe renvoie à l'histoire des idées et à Michel Foucault. Si nous semblons nous éloigner momentanément de la grammaire, nous ne perdons toutefois pas de vue le problème des diminutifs, et cette incursion dans un domaine a priori plus philosophique que grammatical est nécessaire.

D'ailleurs, cette première distinction que nous venons de faire entre d'un côté la philosophie, de l'autre la grammaire, doit d'emblée être repensée lorsque l'on se projette à l'âge classique, où la grammaire, dont la plus représentative est celle de Port-Royal, est une science essentiellement logique. En effet, nous sommes à une époque où la langue est, et c'est là fondamental, vue comme un miroir de la pensée, et où l'on ne sépare pas deux systèmes logiques différents, à savoir le système logique de la pensée et le système logique de la langue, ou plutôt la logique proprement dite et la linguistique. Cette conception de la langue, et de la langue française essentiellement, a été décrite avec beaucoup de pertinence par Michel Foucault dans Les mots et les choses (1966).

Selon Foucault, l'âge classique se caractérise par ce qu'il nomme une « épistémè de la représentation », c'est-à-dire que l'on croit fermement à la nature logique du langage et que ce dernier reflète le monde. Et c'est en effet ce qui apparaît avec netteté lorsqu'on lit les grammaires générales.

L'une des idées fondamentales de ce type de grammaires est que le langage reflète la pensée et son opération principale, le jugement, à travers la proposition, et que le français en particulier, qui suit l'ordre sujet/verbe/objet, est la langue logique par excellence, puisqu'il reflète l'ordre naturel de la pensée, comme aiment à le souligner les grammairiens logiciens (1969: 108):

[...] il n'y a guère de langue qui use moins de ces figures (c'est-à-dire des figures comme l'hyperbate, qui renversent l'ordre sujet/verbe) que la nôtre, parce qu'elle aime particulièrement la netteté, et à exprimer les choses autant qu'il se peut dans l'ordre le plus naturel et le plus désembarrassé, quoiqu'en même temps elle ne cède à aucune en beauté ni en élégance.

On ne s'étonnera pas non plus de trouver des prolongements phonétiques de cette idée d'une langue transparente à travers les théories mimophoniques de certains auteurs comme Court de Gébelin, pour qui le langage est également un reflet phonétique du monde.

Or, puisque le français est une langue parfaitement logique, elle se doit d'être la langue de la science. C'est ce que répètent à l'envi les grammairiens et les philosophes, par exemple Diderot, alors que Rivarol évoque l'universalité de la langue française, universalité justement due à sa structure logique (1930: 264):

Les philosophes l'ont adoptée (la langue française), parce qu'elle [sert de flambeau aux sciences qu'elle traite, et qu'elle] s'accommode également de 
la frugalité didactique et de la magnificence qui convient à l'histoire de la Nature.

Après cette brève description de la théorisation qui est faite de la langue française à l'âge classique, nous pouvons revenir au problème du diminutif.

On voit mieux à présent en effet en quoi ce dernier va à l'encontre de l'idéologie classique de la langue. Le diminutif prête au mot qu'il vient qualifier un caractère approximatif : si je dis «fontelette », je suis dans un au-delà affectif de « fontaine », et « fontelette » représente un lexème bien plus imprécis que le lexème figé en langue, puisqu'il représente un mot de discours subjectif et créé, donc contraire au caractère scientifique de la langue française.

N'oublions pas non plus que le dix-septième siècle est en France un siècle très normatif. On a souvent relevé la pauvreté, au niveau du vocabulaire, des mots employés par Racine ou Corneille. C'est que l'art classique est, on l'a dit également, un art de la litote, qui répondrait assez bien à la définition kantienne de l'art comme maximum de sens avec un minimum de matériau. L'auteur classique emploie des termes lexicalisés en langue, mais très peu de créations discursives, contrairement à son prédécesseur de la Renaissance et à son successeur romantique.

Dans La structure du langage poétique, Jean Cohen écrit (Cohen 1966: 19) :

A notre époque, l'originalité constitue en elle-même un élément de valeur esthétique. A l'époque classique, l'inverse était vrai. La norme était la valeur et l'écart n'était permis qu'à l'intérieur de limites étroites, elles-mêmes garanties par une certaine tradition.

Le diminutif, élément proprement discursif et non codifié en langue, est donc exclu pour toutes les raisons que nous venons d'évoquer : parce qu'il est approximatif et qu'il va à l'encontre de la théorie du langage miroir du monde, parce qu'il est affectif et contraire au caractère scientifique de la langue française (scientifique jusque dans les arts, puisque, rappelons-le, un genre comme la tragédie a pour but la représentation universelle des passions, et doit donc viser au vraisemblable), et parce qu'il est un élément de discours et non de langue, donc une création personnelle alors que l'auteur classique doit s'inscrire dans l'institué de la langue dans un siècle très normatif.

D'où les sermons du père Bouhours contre le diminutif dans Les entretiens d'Ariste et d'Eugène, cités par Bengt Hasselrot, qui ajoute qu'il faut bien tenir compte de l'influence que le Père académicien pouvait avoir sur un auteur comme Racine : «Les fontelette, montagnette, oyselet, ruisselet, qui estoient des délicatesses dans le stile de nos vieux Auteurs, ne se peuvent supporter dans le langage d'aujourd'huy » (Hasselrot 1957: 215); d'où également cette affirmation que nous lisons chez Rivarol (1930: 223) à propos de la langue française de la Renaissance : 
Une foule de poètes s'élevèrent dans son sein, tels que les Jodelle, les Baîf et les Ronsard. Epris d'Homère et de Pindare, et n'ayant pas digéré [les beautés de] ces grands modèles, ils s'imaginèrent que la nation s'était trompée jusque-là, et que la langue française aurait bientôt le charme du grec si on y transportait les mots composés, les diminutifs, les péjoratifs, et surtout la hardiesse des inversions, choses précisément opposées à son génie.

Il suffit de penser que la norme est pour l'époque, selon la règle de Vaugelas, le juste milieu entre le langage des bons auteurs et des courtisans, pour voir que le français qui s'impose est un français sans diminutif, qui n'a plus rien à voir avec le français de la Renaissance de ce point de vue et qui se dégage encore un peu plus des restes synthétiques qu'il gardait pour devenir analytique, et d'un degré d'analyticité qui devient chargé de l'idéologie que nous avons décrite.

Il convient enfin de tenir compte du fait que le français standard ou littéraire est celui qui est enseigné dans les écoles à l'époque où la scolarité devient obligatoire et que les Romantiques, contrairement à ce que l'on aurait pu attendre, n'ont pas récupéré le diminutif (Hasselrot 1957: 217), pour comprendre que ce dernier, devenu absent de la littérature à partir de l'âge classique (la littérature représente la norme scolaire), perd sa vitalité.

On pourrait toutefois nous objecter, et l'on aurait raison, que les Académiciens ne régissent la langue qu'officiellement, et que si le diminutif représentait un besoin expressif, il serait encore présent dans la langue populaire. Or, là encore, il n'en est rien, et il nous semble qu'un deuxième élément vient se superposer à notre première explication, élément d'ordre structural et qui ne contredit en rien le premier argument : le français est une langue plus analytique que les autres langues romanes.

\section{Degrés d'analyticité du français et des autres langues romanes}

Nous allons à présent montrer que le français est probablement la langue romane la plus analytique (nous faisons ici abstraction de certains dialectes qui, séparés de la langue d'origine, ont perdu contact avec elle et se sont développés en se simplifiant énormément; c'est le cas par exemple du portugais de HongKong, qui a perdu toute distinction de genre grammatical et de nombre, et qui exprime le temps de manière très analytique, par périphrase ${ }^{2}$ ). En même temps, nous verrons également l'une des raisons possibles de la conservation du diminutif à l'état spontané dans les autres langues romanes.

Plusieurs éléments nous indiquent que les autres langues romanes (c'est l'espagnol qui nous servira essentiellement de comparaison) sont plus synthétiques que le français. En voici quelques-uns :

\footnotetext{
${ }^{2}$ Voir la description que donne Robert Wallace Thompson dans « O dialecto português de Hongkong» (1961:289-293).
} 
a) Le phénomène d'enclise de la personne dans le verbe. Dans les langues romanes la personne est exprimée de manière synthétique dans la terminaison du verbe, en dehors du pronom : esp. " como », « comes », " come », it " mangio », " mangi », « mangia », etc. Le français a pour sa part recours à un pronom personnel détaché : « je mange », « tu manges », « il mange ». L'espagnol ou l'italien n'utilisent le pronom que pour les cas d'emphase ou pour enlever l'ambigüité du discours.

b) Le système temporel est beaucoup plus développé dans les autres langues romanes qu'en français. Là où le français ne distingue pas sémiologiquement les subjonctifs présent, futur et passé (du moins à l'oral), le portugais les distingue. De même, là où le français ne distingue plus l'aspect global de l'aspect accompli, l'espagnol utilise le prétérit et le passé composé (esp. " Ayer hablé con Lola », fr. « J'ai parlé hier avec Lola »).

c) L'infinitif est utilisé en espagnol et dans d'autres langues romanes pour signifier « le fait de ». Cf. esp. « El haber hablado con ella me complació », mais fr. «Le fait d'avoir parlé avec elle m'a fait plaisir». Le portugais et l'italien ont des tournures pareilles à celle de l'espagnol de ce point de vue (le moyen français avait également cette possibilité d'utiliser l'infinitif substantivé).

d) L'espagnol utilise l'adjectif dérivé beaucoup plus que le français. Citons quelques exemples : "noticiero » (le français a recours à un syntagme adjectival avec préposition : " d'information »), « casadero » (fr. " qui a l'âge de se marier »), « callejero » (fr. « de la rue »), « novelístico » (fr. « du roman »), etc.

e) L'espagnol utilise également beaucoup plus les noms et adjectifs composés que le français : esp. " barbirrubio » (fr. «à la barbe blonde »; cf. également espagnol «barbirrojo » et «barbirrucio »), « boquiancho » (« qui a la bouche large »; cf. également « boquiabierto », " qui a la bouche ouverte », ou « boquiangosto », « qui a la bouche étroite »), « pelirrojo », (« aux cheveux roux »), « pelirrubio » (« aux cheveux blonds »); on retrouve de nombreuses tournures identiques dans d'autres langues romanes d'après Marie-Josée DalberaStefanaggi, et dans une langue comme le corse cette tournure est même créative : « manimozzza» (amputée d'une main), « capellirossu» (aux cheveux roux), « ochjistrambu » (qui louche), à côté de lexicalisations comme «pettirossu », le rouge-gorge, ou «capinera », la mésange à tête noire (DalberaStefanaggi 2002: 46) .

f) L'espagnol recourt à différents suffixes pour exprimer des idées diverses. Ainsi « azo» pour l'idée de « coup de ». Cf. esp. « pepinazo» (« le pétard», mais également un tir puissant au football), «puñetazo » (« coup de poing »), « pucherazo » (« coup de marmite »), « codazo » (« coup de coude »), « vistazo » (« coup d'oeil »), etc. L'espagnol a également le suffixe « ada » pour rendre cette idée : « colmillada » (fr. « coup de dent »), « agarrada » (fr. " prise de bec », d'où idée de coup symboliquement), « ojeada » (« coup d'oeil »), etc. 
On voit par ces quelques remarques qui devraient être complétées que l'espagnol et les autres langues romanes sont plus synthétiques que le français, malgré la perte tardive des cas dans cette dernière langue. Il n'est pas étonnant dès lors que le diminutif, qui est un élément synthétique, ait perdu toute sa vitalité en français moderne et demeure essentiellement lexicalisé (à l'exception de « ette » dont nous avons dit qu'il était encore parfois un peu créatif).

Or, si nous quittons un moment les langues romanes pour aller voir une autre famille de langues, à savoir les langues germaniques, il nous semble que le même phénomène s'observe entre l'allemand et l'anglais. Alors que l'allemand a conservé des cas et des suffixes diminutifs ( «lein» et «chen»), l'anglais, qui est sans doute la plus analytique des langues issues de l'indo-européen, n'a jamais recours à des appréciatifs ${ }^{3}$, tout juste à l'adjectif « little». Il en va de même pour une langue comme le suédois, langue scandinave sans cas qui est à la base, comme l'anglais, une langue germanique, et qui a développé une appréciation analytique avec l'adjectif « lilla ». Les faits de linguistique comparée nous éclairent donc sur un phénomène de linguistique romane.

\section{Références bibliographiques}

ARNAULD, Antoine et Claude LANCELOT (1969): Grammaire générale et raisonnée de Port-Royal, avec les remarques de Ch. Duclos, introduction de Michel Foucault. Paris: Republications Paulet.

COHEN, Jean (1966): Structure du langage poétique. Paris: Flammarion.

DALBERA-STEFANAGGI, Marie-José (2002): La langue corse. Paris : PUF.

FOUCAULT, Michel (1966): Les mots et les choses. Paris: Gallimard.

HASSELROT, Bengt (1957): Études sur la formation diminutive dans les langues romanes. Uppsala: Acta Universitatis Upsaliensis.

HASSELROT, Bengt (1972): Étude sur la vitalité de la formation diminutive française au XXe siècle. Uppsala: Acta Universitatis Upsaliensis.

MendozA, José (2008): «Bolivia », in El español en América. Contactos lingüisticos en Hispanoamérica, pp. 213-236. Barcelona: Editorial Ariel.

REAL ACADEMIA ESPAÑOLA (2009): Nueva gramática de la lengua española. Morfología, Sintaxis, tome 1. Madrid: Asociación de Academias de la Lengua Española.

REAL ACADEMIA ESPAÑOLA: CREA

RIVAROL, Antoine (1930): De l'universalité de la langue française, texte établi et commenté par Th. Suran. Paris: H. Didier.

THOMPSON, Robert Wallace (1961): «O dialecto português de Hongkong ». Boletim de filologia portuguesa 19: 289-293.

\footnotetext{
${ }^{3}$ Tout au plus peut-on relever des formations de type : « aunts » qui donnes « aunties ».
} 